\title{
Real-time Color Detection System using Custom LSI for High-Speed Machine Vision
}

\author{
Junichi Akita ${ }^{1}$ \\ Dept. of Elec. and Comp. Eng., Kanazawa University \\ 2-40-20 Kodatsuno, Kanazawa, Ishikawa, 920-8667 Japan
}

\begin{abstract}
The quantity of image information is very large, and this is why it is difficult to process them in real time, such as video frame rate. In this paper we demonstrate the developed real-time color detection system for the vision system of RoboCup Small-Size League. The pixel's color information is converted to HSV color system at first, and judged whether it has the color information of target. The detected information is converted to gray scale NTSC signal to be captured by PC, which is faster than the full color frame grabber.
\end{abstract}

\section{Introduction}

The quantity of image information is very large, and this is why it is difficult to process them in real time, such as video frame rate. Pixel information from video camera is transfereed from the upper-left corner of image to the lower-right corner serially, and the color detection of pixel can be processed only according to its color information, not those of neighbor pixels.

In this paper, we describe the real-time color detection system for the vision system of RoboCup Small-Size League using specially designed LSI. We also describe the real-time color conversion system from RGB to HSV employing pipelined operation of look-up table using ROM. The original color image is fed into the developed system, containing color conversion system and color detection system, and then the gray scale image generated by color detection results comes out in 600ns, and this system can be regarded as a 'filter' of image.

The post-color-detection processes, such as labeling, recognition, are processed for these detected 'gray' image, which is much easier to process in real time than the case of both color detection and other processes are executed by software.

\section{Elements of Color Detection System}

Figure 1 shows the structure of the developed real-time color detection system for vision system of RoboCup Small-Size League. The video signal from color CCD camera in NTSC format is converted to RGB signal at first, and then 


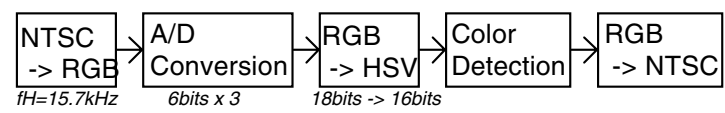

Fig. 1. Structure of real-time color detection system

converted to digital signal of $6 \times 3$ bits. The digitized RGB signal is converted to the pair of HSV (Hue, Saturation, and Value), and then judged whether it is a target color or not by color detection circuit. The color detection results are converted to gray scale NTSC video signal again, which PC can easily capture by frame grabber. The detection result per pixel comes out $600 \mathrm{~ns}$ after original pixel data is fed in, which can be regarded as 'simultaneously,' and the processed image is generated by passing a kind of 'filter.'

\subsection{Color conversion methodology}

The video signal in NTSC format from color CCD camera is at first converted to RGB signal, with the horizontal synchronous frequency of $15.7 \mathrm{kHz}$. It may be usual to express the color information of each pixel by the pair of RGB, that is the intensities of red, green, and blue, that are intrinsically acquired by CCD color imager. It will be a better way to express the color information of pixel by the pair of HSV, since the pair of HSV is more invariant than the pair of RGB for the change of lighting condition.

The conversion rule of the pair of RGB to the pair of HSV is a simple, but a non linear function. One idea to execute this conversion is to calculate this simple, but non linear conversion rule for each pixel. This procedure can be easily implemented by software program, but it needs much processing time. For example, the pixel clock cycle time is about 100ns in NTSC video signal, and the conversion from RGB to HSV should be completed in 100ns for real-time process, that is almost impossible by employing software process.

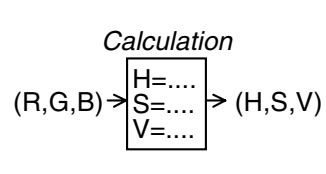

(a)

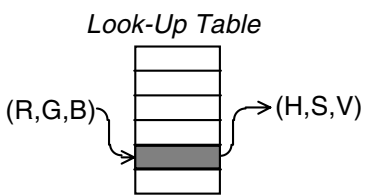

(b)

Fig. 2. Two methodologies to convert from RGB to HSV, (a)calculation per pixel, (b)employing look-up table

The other idea to execute this RGB to HSV conversion is to employ lookup table of RGB to HSV. The HSV values should be calculated for all case of possible RGB values once, and they are stored in look-up table. In conversion 
process, the input RGB values is used to indicate the according point of this look-up table, and the according result in this look-up table will be obtained much faster than to calculate it, as shown in Fig.2.

The look-up table can be easily implemented by ROM (Read Only Memory), where input RGB values are used as its address, and output HSV values are read out as the data in its address. Assuming the number of bits of RGB values as $6 \times 3$ bits, the total address space of ROM is equal to $2^{18}=256 \mathrm{~K}$, which is reasonable to implement by using popular ROM IC. If we employ $8 \times 3$ bits for RGB values, the needed address space is $2^{24}=16 \mathrm{M}$, which will need more than 8 chips of ROM for one conversion look-up table bank.

\subsection{Color detection criteria}

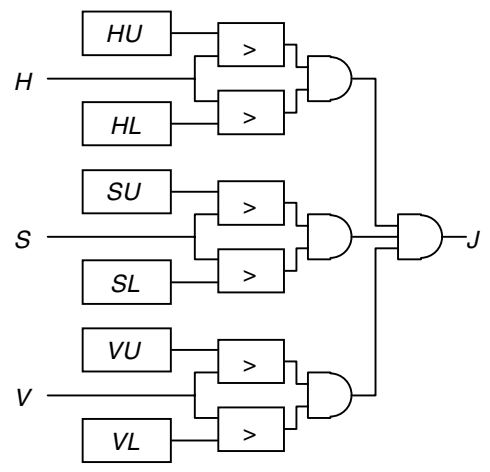

Fig. 3. Structure of color detector

The simple color detection criteria we have employed here is to detect all of hue, saturation, and value stay in the desired range, which is expressed as follows,

$$
\begin{aligned}
J= & \left(H_{L}<H\right) \text { and }\left(H<H_{U}\right) \\
& \text { and }\left(S_{L}<S\right) \text { and }\left(S<S_{U}\right) \\
& \text { and }\left(V_{L}<V\right) \text { and }\left(V<V_{U}\right),
\end{aligned}
$$

where $J$ is the judgement flag of detection, $H, S$ and, $V$ are the value of hue, saturation, and value, respectively, $H_{L}$ and $H_{U}$ are the preset lower and upper thresholds of hue, respectively, and so on.

In our developed system, the number of bits of each component is 6 for hue, 5 for both saturation and value, and the comparison operation should be executed in the accuracy of these bits. They are also needed the register where the upper and lower thresholds of each component are stored.

The whole circuit structure of the color detector is shown in Fig.3. 


\subsection{Design of color detection chip}

We have designed the color detection chip using CMOS $1.2 \mu \mathrm{m}$, double metal, double poly technology through LSI fabrication service of VDEC[VDEC, 1998] ${ }^{1}$.

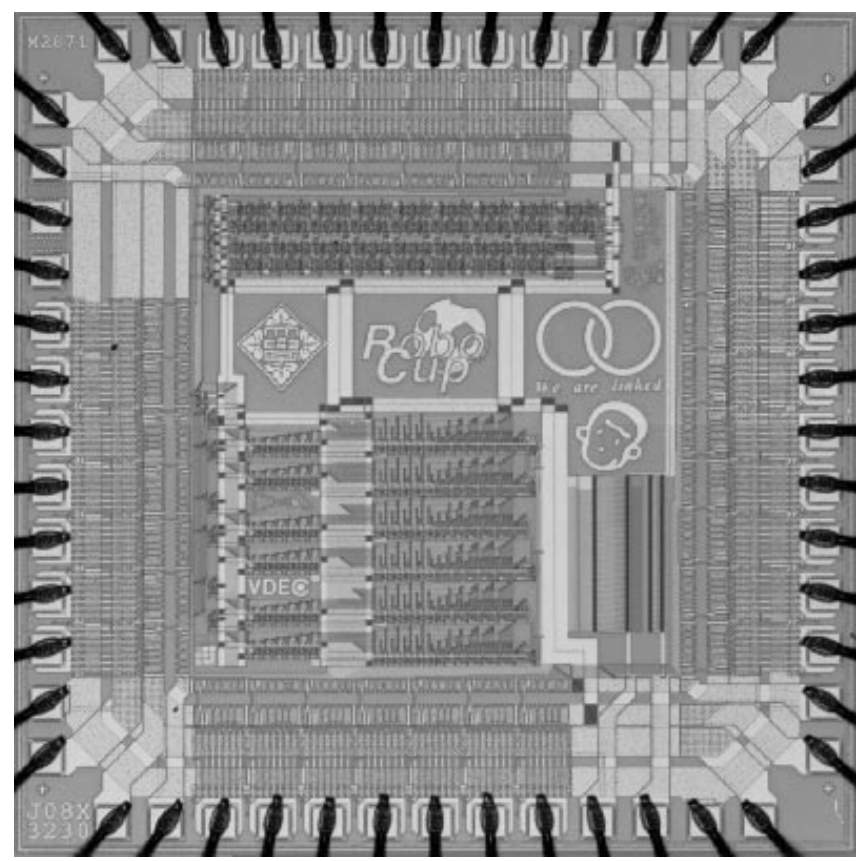

Fig. 4. Designed real-time color detection chip

The designed color detection chip consists of three blocks; 6bits magnitude comparators and 6bits registers and logic AND gates. We have designed them manually using layout editor, and gathered them in one chip with their interconnections drawn by layout editor Fig.4 shows the photograph of fabricated chip. The chip size is $2.3 \mathrm{~mm} \times 2.3 \mathrm{~mm}$, and the number of transistors is 2,174 . The measured operation speed of this chip is $11.2 \mathrm{~ns}$, which is fast enough for color detection in one pixel clock cycle, about 100ns.

The operation of color detection can be processed only according to the information of one pixel, and the detection circuit does not need to have frame memory which stores the whole image, and this is why the designed circuit consists of small size transistors and operates fast enough.

${ }^{1}$ The VLSI chip in this study has been fabricated in the chip fabrication program of VLSI Design and Education Center(VDEC), the University of Tokyo with the collaboration by Nippon Motorola LTD., Dai Nippon Printing Corporation, and KYOCERA Corporation. 


\section{Developed Color Detection System}

The developed real-time color detection system consists of two blocks; color conversion block and color detection block.

\subsection{Developed color conversion block}

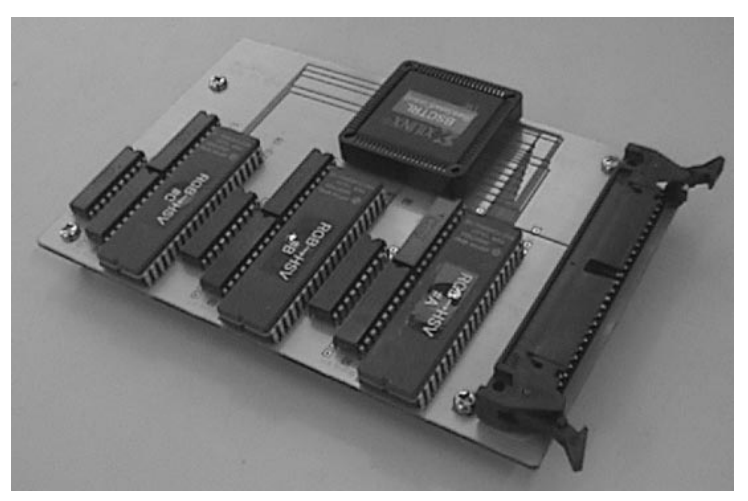

Fig. 5. Developed real-time RGB to HSV conversion board

Figure 5 shows the developed circuit board to convert pixel color information from RGB to HSV. The acquired RGB signal of pixel is 18bits; 6bits for red, green, and blue respectively. We have employed the total 18 bits for RGB values in order to reduce the number of ROMs for look-up table in reasonable level.

The converted HSV information is assumed to be composed of $6 \mathrm{bits}$ of hue, $\mathrm{H}$, 5bits of saturation, S, and 5bits of value, V. The conversion look-up table is implemented by $4 \mathrm{Mbits}$ EPROM of $27 \mathrm{HC} 4096$, which consists of $16 \mathrm{bits} \times 2^{18}$. The data for each memory cell should contain according values of HSV, and this contains $6+5+5=16$ bits.

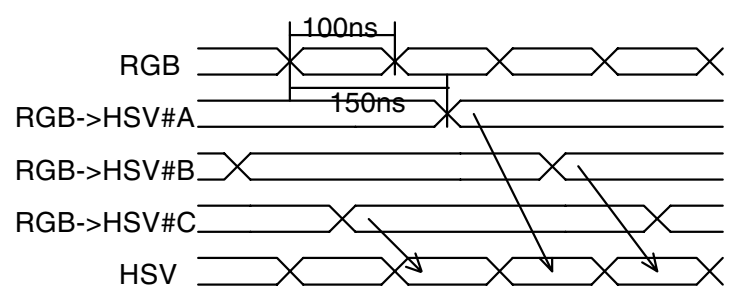

Fig. 6. Operation timing of RGB to HSV conversion

The access time of the ROM we employed is $150 \mathrm{~ns}$, which is longer than 
the cycle time of pixel clock, about 100ns. In order to maintain the average processing time per pixel within 100ns, we have employed three banks of ROM, and they are used in order, which is a kind of pipeline architecture, shown in Fig.6. The RGB is passed to ROM of bank\#A, and the result will come out after 150ns. The RGB data of the following pixel is passed to ROM of bank\#B, and the next to bank\#C, and at this time the conversion result of bank\#A is obtained, and passed to output latch of final HSV conversion result, and the result of bank\#C will be obtained in the next cycle, and so on.

The control of this pipeline operation of three ROM banks is implemented by programmable logic (Complex Programmable Logic Device; CPLD) of XC95108 by Xilinx Corp[Xilinx, 1998], with using ABEL (Advanced Boolean Expression Language) as function description language.

\subsection{Developed color detection block}

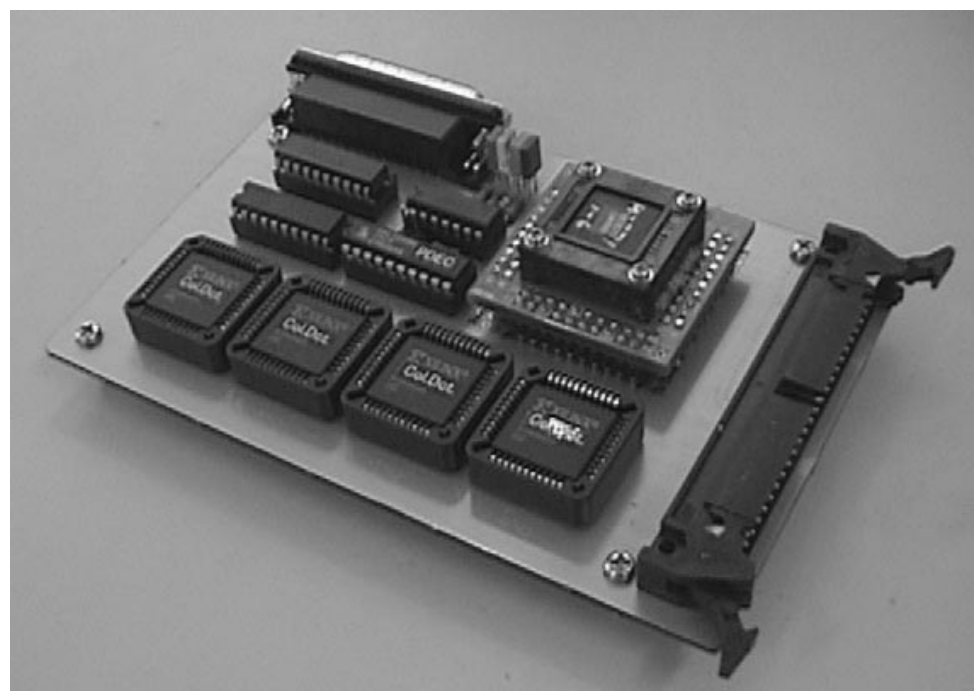

Fig. 7. Developed real-time color detection board

Figure 7 shows the developed color detection block including the designed chip above. The HSV signal of each pixel is passed to four color detection chip at the same time, in order to detect four colors simultaneously. The four outputs of all color detection chip are gathered again, in order to generate the gray scale image according to the detected color using 4 bits priority encoder. For example, it generates 'white' pixel for input of 'orange' pixel (that is the color of ball), 'bright gray' for 'blue' (that is the color of robots' marker), 'medium gray' for 'pink' (that is the color of robots' second marker to detect their directions), and 'dark gray' for 'yellow' (that is the color of enemy robots' marker). The registers 
of each color detectors which hold the upper and lower thresholds are set by external controller, it is set by the keyboard in this system. These thresholds are decided manually, in order to the target color can detect independently.

The generated gray scale image based on color detection is converted to NTSC video signal, which frame grabber of PC can capture. It will be a best way to send the detection results to PC by digital data, and we are currently under development of this block.

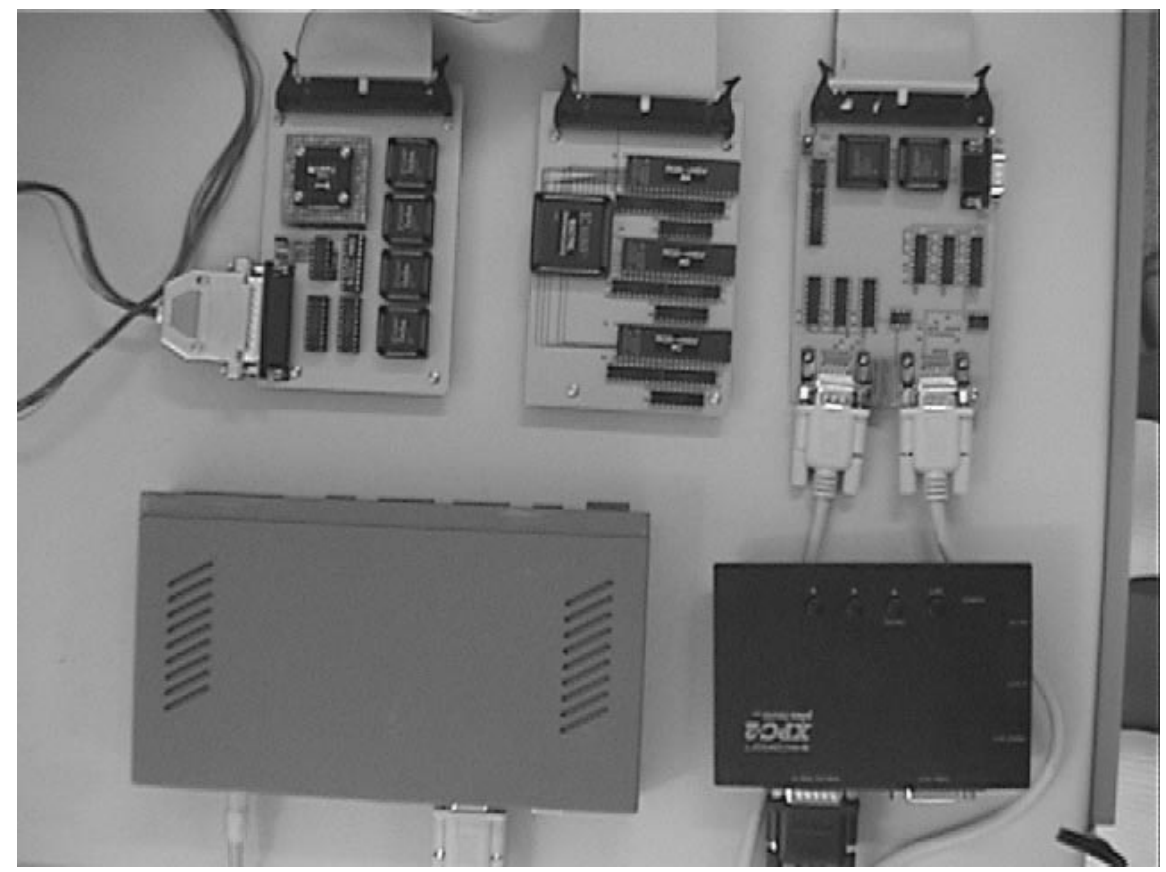

Fig. 8. Whole of the developed real-time color detection system

Figure 8 shows the whole of the developed real-time color detection system, including NTSC to RGB converter, clock generator and A/D, D/A conversion blocks, the color conversion block, the color detection block, and RGB to NTSC converter.

\section{Experimental Results}

We have carried out the experiments for color detection. Sample color images are captured by CCD color video camera, and this signal is fed into the developed system. The color detection results in gray-scale image is again captured by PC. Figure 9 shows one of the experimental results. The input image of Fig.9(a), where the text of 'Red' is written in red ink, 'Blue' in blue ink, and so on, is 


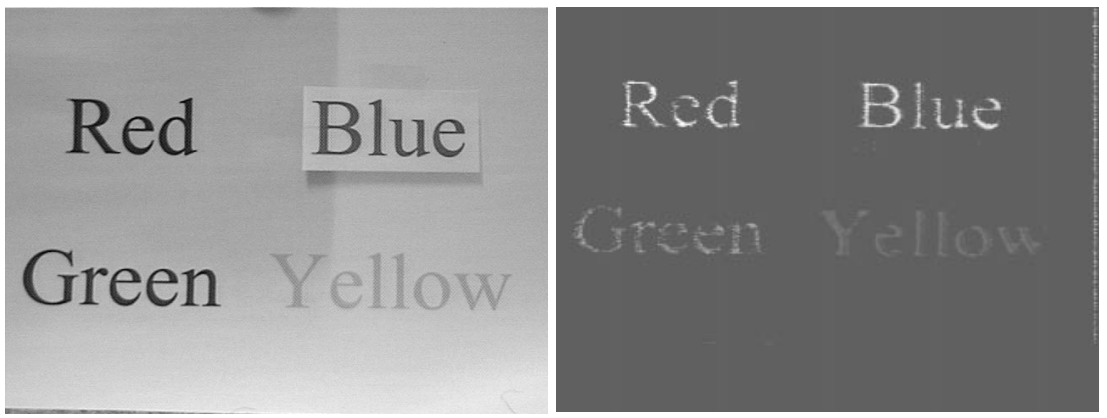

Fig. 9. The experimental result of color detection using the developed system, (a)original color image, (b)generated gray image based on color detection

processed by the developed system with the sequence of Fig.1. The generated gray image based on color detection is shown in Fig.9(b). We can recognize some lack of component pixels containing in each text, but it will be improved by tuning the threshold values of each color, and reducing the noise in $A / D$ and $\mathrm{D} / \mathrm{A}$ conversion block. It will be an another way to increase the number of bits of each color values, but it will result in the increase of the size of ROM. It will be reasonable to employ $7 \times 3$ bits for each color values, which will result in $2^{21} \times(7+7+7) \simeq 16$ Mbytes in one bank of look-up table.

\section{Conclusion}

In this paper, we have described the developed the real-time color detection system for the vision system of RoboCup Small-Size League. The color image is fed into this system, and the result of color detection is automatically generated in NTSC video format, which can be easily captured by gray-scale frame grabber, that is faster than full-color frame grabber. This system is expected to be effective to reduce the load of image processing system; it can process just after color detection.

\section{Acknowledgements}

This study is aided by "Yugen Club Found" of Japanese Association for Mathematical Science[JAMS, 1998].

\section{References}

[Xilinx, 1998] http://www.xilinx.com

[VDEC, 1998] http://www.vdec.u-tokyo.ac.jp

[JAMS, 1998] http://www . yugen.org 